\title{
Condition Analysis of Steam Turbine DEH Control System based on Data Fusion
}

\author{
Zhenhe Wang \\ College of Sciences, Hebei University of Science and Technology, Shijiazhuang, Hebei \\ 050018, China
}

e-mail:0313wzh@163.com

Keywords-DEH control system; condition analysis; data fusion

Abstract-Aiming at the problem that the system of digital electro-hydraulic(DEH) regulation system is complex and difficult to analyze its condition, a method based on characteristic extraction and information fusion is proposed. By making RCM analysis, the indexes for quantitative risk evaluation of fault modes are determined, and the fuzzy rule base for risk evaluation is built. Using fuzzy inference, the system fault modes are ranked according to their risk level. Then, the condition characteristic parameters are extracted according to the pivot fault modes. Using the extracted characteristic parameters, an information fusion method based on evidence theory is put forward to evaluate the system's condition. It is shown by the instance that this method is feasible and effective and the condition analysis results can be used as a support for next maintenance decisions.

\section{INTRODUCTION}

With the rapid developing of electric power industry in our country, the maintenance mode is changing from planned maintenance to condition-based maintenance. The pivotal task of condition-based maintenance is to evaluate the equipment condition synthetically and arrange maintenance times and items reasonably. DEH regulation system is complex and includes many components. Meanwhile, the relations between subsystems have strong relationships. In condition analysis, reasonable condition characteristic extraction method and accurate condition evaluation model are two pivotal aspects. But, in most methods ${ }^{[1]}$, condition characteristics are extracted just using qualitative analyzing and expert opinion, and the condition evaluation model is just linear integration. The accuracy of condition evaluation results is weaken greatly. So based on RCM analysis, fuzzy theory and evidence theory, a method of condition analysis is put forward for DEH system.

In the paper, take DEH regulating system as an example, all subsystems are analyzed in RCM method based on system division and the fault modes and their risk evaluation indexes are determined. Then based on fuzzy theory, the risk levels of all fault modes are evaluated quantificationally using fuzzy inference method and the characteristics are extracted by analyzing the fault modes with higher risk levels. Finally, the system condition is analyzed by information fusion arithmetic based on D-S theory.

\section{RCM ANALYSIS ON POWER STATION EQUIPMENT}

A. System division of DEH regulating system

According to the principle of function correlation, the system division is shown in Fig.1 


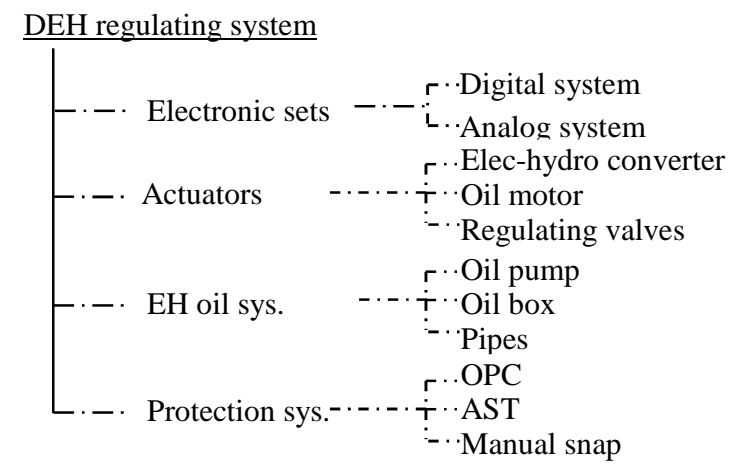

Fig.1 The division of DEH regulating system

\section{B. FMEA on DEH regulating system}

In the process of fault mode and effective analysis, the possible fault modes and their effects of subsystem or components are listed in the form of table $\mathrm{e}^{[2,3]}$. In the same time, the risk evaluation indexes of fault modes are also determined. Based on the actual operation condition of DEH regulating system, the components prone to fail are selected to be analyzed.The possible fault modes of the components are determined. In Tab.1, the fault modes and their risk evaluation factor values of oil motor are listed.

\begin{tabular}{ccccc} 
Tab.1 Oil motor' failure modes and linguistic descriptions \\
\hline FM no. & Failure modes & Severity & Occ Freq. & Dete. \\
\hline F1 & Inlet jammed & Mod & Vlow & Mod \\
F2 & Outlet jammed & Mod & Low & Low \\
F3 & Spring rapture & Mod & Low & Mod \\
F4 & Unfit preload & Mod & Low & Low \\
F6 & Piston jammed & Mod & High & Low \\
F7 & Piston wear & Mod & High & Low \\
F8 & Sealing ring bad & Mod & Mod & Mod \\
F9 & LVDT break & Mod & Low & High \\
\hline
\end{tabular}

\section{Quantitative indexes of failure mode risk analysis}

During the risk evaluating of failure mode, the 3 indexes of failure severity, probability of occurrence and detectability should be considered. The evaluation criterions of the there indexes are shown in table 2, table 3 and table 4 . In addition, the risk evaluation index of failure mode is shown in table 5 .

Tab.2 The scale for severity

\begin{tabular}{cc}
\hline Severity & Rating \\
\hline Very low & 1 \\
Low & 2 \\
& 3 \\
Moderate & 4 \\
& 5 \\
High & 6 \\
Very high & 7 \\
& 8 \\
\end{tabular}


Tab.3 The scale for probability of occurrence

\begin{tabular}{ccc}
\hline Probability of occurrence & Failure rate(day) & Rating \\
\hline Very low & $<1: 20000$ & 1 \\
Low & $1: 20000$ & 2 \\
& $1: 10000$ & 3 \\
Moderate & $1: 2000$ & 4 \\
& $1: 1000$ & 5 \\
High & $1: 200$ & 6 \\
& $1: 100$ & 7 \\
Very high & $1: 20$ & 8 \\
& $1: 10$ & 9 \\
& $1: 2$ & 10 \\
\hline
\end{tabular}

Tab. 4 The scale for detectability

\begin{tabular}{ccc}
\hline Detectability & Probability of detection(\%) & Rating \\
\hline Very high & $85-100$ & 1 \\
High & $75-85$ & 2 \\
& $65-75$ & 3 \\
Moderate & $55-65$ & 4 \\
& $45-55$ & 5 \\
Low & $35-45$ & 6 \\
& $25-35$ & 7 \\
Very low & $15-25$ & 8 \\
& $5-15$ & 9 \\
& $0-5$ & 10 \\
\hline
\end{tabular}

Tab.5 The scale for risk evaluation

\begin{tabular}{llllll}
\hline Risk & None & Elow & Vlow & Rlow & Low \\
\hline Rating & 1 & 2 & 3 & 4 & 5 \\
\hline Risk & Mod & High & Rhigh & Vhigh & Ehigh \\
\hline Rating & 6 & 7 & 8 & 9 & 10 \\
\hline
\end{tabular}

\section{RISK ANALYSIS ON FAILURE MODES BASED ON FUZZY INFERENCE}

\section{A. Fuzzy membership function ${ }^{[4,5]}$}

Depending on the table $2 \sim 5$ and the experiences of Depending on the table $2 \sim 5$ and the experiences of domain experts, the fuzzy membership functions for the severity, the probability of occurrence, the detectability and risk of failure mode is set up, and shown in figure2 5.

In the figures, the scope of abscissa is between 1 and 10. Using figures of fuzzy membership function, the corresponding fuzzy membership value can be gained when input the evaluation value of a certain index. The value of a certain index can be decided by the there evaluation criterion tables.

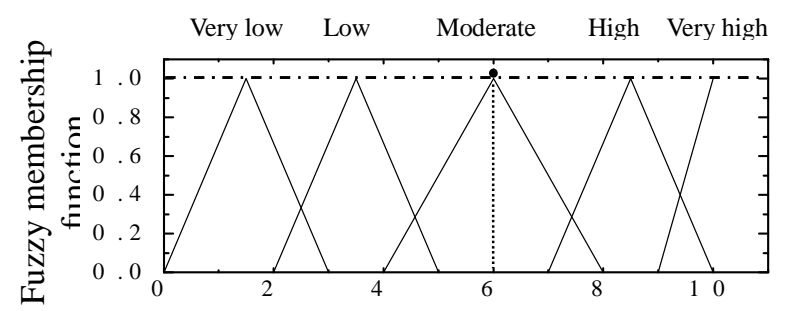

Fig. 2 The severity

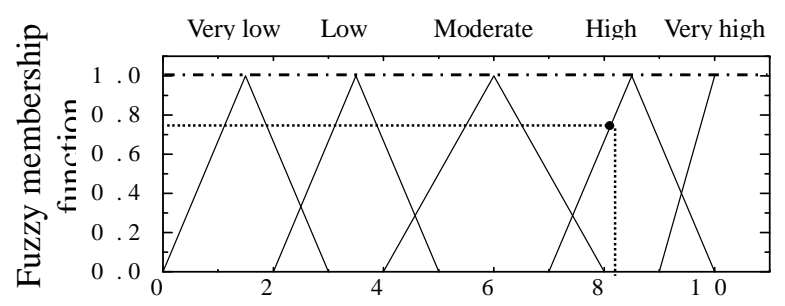

Fig. 3 The probability of occurrence 


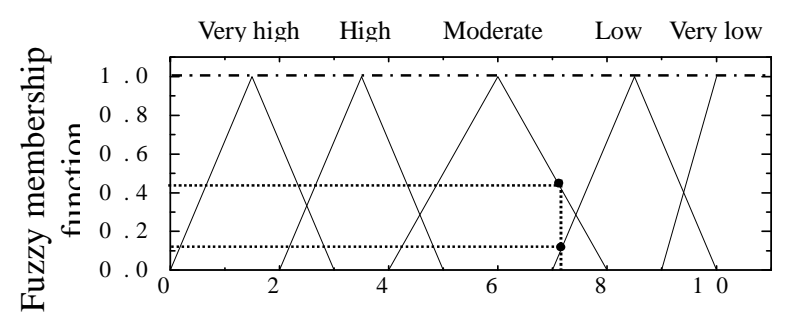

Fig. 4 The detectivity

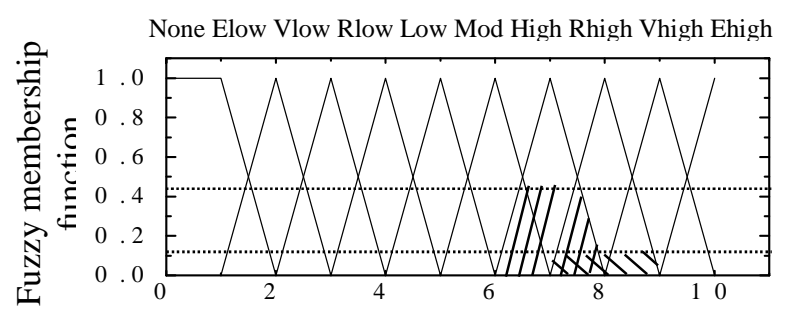

Fig. 5 The risk level

\section{B. Fuzzy rule base development}

Fuzzy inference systems are knowledge-based or rule-based systems constructed from human knowledge in the form of fuzzy IF-THEN rules. In fuzzy inference systems, the rule base describes the synthetic risk caused by input variables with different combination. The rules usually are described by natural language, and in the form of IF-THEN. The IF-THEN rule consists two parts, one is precondition which can compares with input, the other is the result under the precondition. In this paper, the input of rule base is severity, probability of occurrence and detectability, the output is a linguistic variable, that is, the criterion of fault mode risk evaluation.

\section{Principle of fuzzy inference}

The min-max fuzzy inference form is used in the paper $[6,7]$, there are 3 important formulus as follows.

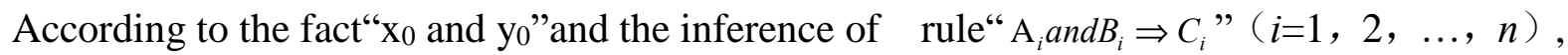
the fuzzy membership grade of $C_{i}^{\prime}$ can be got using formula (1)

$$
\mu_{C_{i}^{\prime}}\left(z_{i}\right)=\mu_{A_{i}}\left(x_{0}\right) \wedge \mu_{B_{i}}\left(y_{0}\right)
$$

Suppose a certain fact $x_{1}, y_{1}$, from $m$ rules, a same inference result $C_{i}^{\prime}(i=1,2, \mathrm{~L}, m)$ is obtained. The $\mathrm{m}$ rules correspond to $m$ fuzzy membership $\mu_{C_{i 1}^{\prime}}, \mu_{C_{i 2}^{\prime}}, \mathrm{L}, \mu_{C_{i m}^{\prime}}$. The fuzzy membership grade of result $C_{i}^{\prime}$ can be calculated using formula (2)

$$
\mu_{C_{i}^{\prime}}\left(z_{i}\right)=\mu_{C_{i 1}^{\prime}}\left(z_{i}\right) \vee \mu_{C_{i 2}^{\prime}}\left(z_{i}\right) \vee \mathrm{L} \vee \mu_{C_{i m}^{\prime}}\left(z_{i}\right)
$$

According to the fact $x_{0}, y_{0}$, the fuzzy membership grade of $C_{1}^{\prime}, C_{2}^{\prime}$ is $\mu_{C_{1}^{\prime}}\left(z_{1}\right), \mu_{C_{2}^{\prime}}\left(z_{2}\right)$. Then, a method of weighted averaging can be used to get the numeral value of the fuzzy result $C^{\prime}\left(C_{1}^{\prime}, C_{2}^{\prime}\right)$, and the representative value $z_{0}$ is obtained.

$$
z_{0}=\sum_{i=1}^{2} z_{i} \mu_{C_{i}^{\prime}}\left(z_{i}\right) / \sum_{i=1}^{2} \mu_{C_{i}^{\prime}}\left(z_{i}\right) \quad z_{i} \in Z
$$

Thus, using formula (1) (3), the fuzzy inference from fact " $x_{0}$ and $y_{0}$ " to the result representative point $z_{0}$ can be realized. 


\section{CONDITION ANALYSIS BASED ON INFORMATION FUSION}

\section{A. Decision tree information fusion model}

Typical decision tree information fusion model is as follow ${ }^{[8]}$.

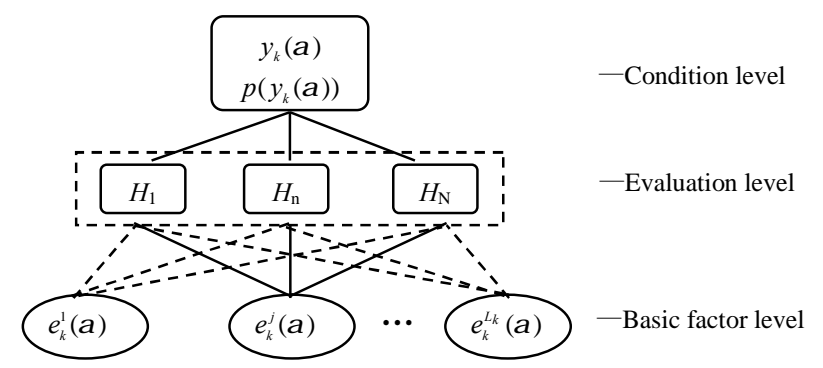

Fig.6 A representative decision tree information fusion model

In evaluation level, $H_{n}$ is called an evaluation grade $(n=1, \mathrm{~L}, N)$. A set of evaluation grades for condition attribute $y_{k}$ is denoted by

$$
H=\left\{H_{1}, \mathrm{~L}, H_{n}, \mathrm{~L}, H_{N}\right\}
$$

where $N$ is the number of evaluation grades. $H_{1}$ and $H_{N}$ are set to be the worst and the best grades, respectively, and $H_{n+1}$ is preferred to $H_{n}$.

The qualitative evaluation is difficult to give, as it is subjective and sometimes incomplete. In order to quantify these evaluation grades and eventually to quantify subjective judgments with uncertainty, the concept of preference degree was introduced. A preference degree takes values from the close interval $[-1,1]$, the set of evaluation grades may thus be quantified by

$$
p\{H\}=\left[p\left(H_{1}\right), \mathrm{L}, p\left(H_{n}\right), \mathrm{L}, p\left(H_{N}\right)\right]^{T}
$$

where $p\left(H_{n}\right)$ is the scale of $H_{n}$ and satisfies the following basic conditions:

$$
p\left(H_{1}\right)=-1, \quad p\left(H_{N}\right)=1
$$

$$
p\left(H_{n+1}\right)>p\left(H_{n}\right), \quad n=1, \mathrm{~L}, N-1
$$

In basic factor level, the factor set connecting with condition attribute evaluation is as follow:

$$
E_{k}=\left\{e_{k}^{1}, e_{k}^{2}, \mathrm{~L}, e_{k}^{L_{k}}\right\}
$$

where $e_{k}^{i}\left(i=1, \mathrm{~L}, L_{k}\right)$ denotes the factors affecting the evaluation on $\mathrm{y}_{\mathrm{k}}$, the evaluation of $e_{k}^{i}$ can be determined by connected condition characteristic parameters, $e_{k}^{i}=e_{k}^{i}(\alpha)$, A larger preference degree value is interpreted as a higher evaluation grade. So the preference degree for the state of an condition attribute $\mathrm{y}_{\mathrm{k}}$ through the direct evaluations of the relevant factors $e_{k}^{i}$ can then be generated and integrated by using the D-S evidence theory presented follow.

\section{B. Condition analysis arithmetic based on information fusion}

D-S evidence theory is a kind of information fusion classification arithmetic based on statistic, the Dempster rule combining multiple information is as follow:

In the decision tree information fusion model, evaluation grade $H_{n}$ can be regarded as a basic hypothesis of D-S evidence theory, factor $e_{k}^{i}$ as a piece of evidence, and a basic probability assignment may be obtained from a confidence degree. All of the evaluation grades in $H$ are defined as distinct grades. With this in mind, the frame of discernment may be defined by

$$
\Theta=H=\left\{H_{1}, \mathrm{~L}, H_{n}, \mathrm{~L}, H_{N}\right\}
$$

Let $M\left(H_{n} / e_{k}^{i}(\alpha)\right)$ express a basic probability assignment to which $e_{k}^{i}$ supports a hypothesis that the state of $\mathrm{y}_{\mathrm{k}}$ is conform to $H_{n}$, also let $\beta\left(e_{k}^{i}(\alpha)\right)$ be a confidence degree to which the decision maker considers that the state of $e_{k}^{i}$ is confirmed. 
If there is only one factor $e_{k}^{i}$ in $E_{k}, m\left(H_{n} / e_{k}^{i}(\alpha)\right)$ should be equal to $\beta\left(e_{k}^{i}(\alpha)\right)$; if there are multiple factors in $E_{k}$, then:

$$
M\left(H_{n} / e_{k}^{i}(\alpha)\right)=\lambda_{k}^{i} \beta\left(e_{k}^{i}(\alpha)\right)
$$

in which, $\lambda_{k}^{i}$ are the weights of all factors $e_{k}^{i}$ in $E_{k}$.

After gained the basic probability assignments, the overall probability assignment can be calculated by using the following evidence reasoning arithmetic.

Define a factor subset $e_{L_{k}(i)}(\alpha)$ and a combined probability assignment $M M_{r i}^{C}(\alpha)$ as follow:

$$
\begin{array}{r}
e_{L_{k}(i)}(\alpha)=\left\{e_{k}^{1}(\alpha), \mathrm{L}, e_{k}^{i}(\alpha)\right\}, \quad 1 \leq i \leq L_{k} \\
M M_{\mathrm{r}, i}^{C}(\alpha)=M\left(\frac{C}{e_{L_{k}(i)}(\alpha)}\right)=M_{r i}^{C}(\alpha)
\end{array}
$$

Then, initial condition:

$$
M M_{r, 1}^{n}=M_{r, 1}^{n} ; \quad M M_{r, 1}^{H}=M_{r, 1}^{H}
$$

iterative formula:

$$
\begin{array}{r}
M M_{r, i+1}^{n}=K_{r, i+1}\left(M M_{r, i}^{n} M_{r, i+1}^{n}+M M_{r, i}^{n} M_{r, i+1}^{H}+M M_{r, i}^{H} M_{r, i+1}^{n}\right) \\
M M_{r, i+1}^{H}=K_{r, i+1} M M_{r, i}^{H} M_{r, i+1}^{H}
\end{array}
$$

in which, $K_{r, i+1}=\left[1-\sum_{t=1}^{N} \sum_{s=1, s \neq t}^{N} M M_{r, i}^{t} M_{r, i+1}^{s}\right]^{-1}$,

$$
i=1, \mathrm{~L}, L_{k}-1, \quad r=1, \mathrm{~L}, R
$$

Then the overall preference degree can be calculated as follow:

$$
p_{r k}=p\left(y_{k}\left(\alpha_{r}\right)\right)=\sum_{n=1}^{N} M M_{r, L_{k}}^{n} p\left(H_{n}\right)+M M_{r, L_{k}}^{H} p(H)
$$

where, $p(H)=\sum_{n=1}^{N} \frac{p\left(H_{n}\right)}{N}$.

\section{Condition analysis example of the DEH regulation system}

Took a 300MW steam turbine unit DEH regulation system as example. Using the results of section 3, the condition characteristic parameter values of a certain running time is listed as tab.9.

In this example, the condition space is defined as follow:

$$
\mathrm{H}=\left\{\mathrm{H}_{1}, \mathrm{H}_{2}, \mathrm{H}_{3}, \mathrm{H}_{4}, \mathrm{H}_{5}\right\} \quad=\{\text { serious, fault, general, good, best }\}
$$

Tab.6 Condition characteristic parameter values of DEH regulation system

\begin{tabular}{lllll}
\hline & Parameter names & $\begin{array}{c}\text { Threshold ; } \\
\text { normal values }\end{array}$ & $\begin{array}{c}\text { Actual } \\
\text { values }\end{array}$ & units \\
\hline \multirow{2}{*}{$\begin{array}{llll}\text { Ratio of flutter } \\
\text { Oil pressure difference }\end{array}$} & $>0.5 ; 1$ & 0.84 & - \\
& $<1 ; 0.2$ & 0.42 & $\mathrm{Mpa}$ \\
between in and out & & & \\
ors & Down oil pressure of oil & $<1.5 ; 1$ & 1.16 & - \\
& motor & $<5 ;-$ & 1.6 & - \\
& Worn scraps in oil & $<0.2 ; 0$ & 0.042 & - \\
& Flux warp of valve & $<1.5 ; 1$ & 1.16 & - \\
\hline \multirow{3}{*}{$\begin{array}{l}\text { EH oil } \\
\text { system }\end{array}$} & EH oil pump swing & $<50 ;-$ & 16 & $\mu \mathrm{m}$ \\
& EH oil pump current & $<30 ; 21$ & 25.5 & $\mathrm{~A}$ \\
& Oil temperature & $<57 ; 43$ & 46 & ${ }^{\circ} \mathrm{C}$ \\
\hline Protect & EH oil main pipe pressure & $11-16 ; 14.5$ & 13 & $\mathrm{Mpa}$ \\
ion sys & OPC oil pressure & $>1 ; 1.96$ & 1.78 & $\mathrm{Mpa}$ \\
\hline
\end{tabular}

After making unitary treatment and fuzzy transform, the results are listed in tab.10, which 
are used as the input for system condition analysis.

Tab.7 Input data for system condition analysis

\begin{tabular}{lllcc}
\hline Parameter names & Weight & $\mathrm{H}_{1}$ & $\mathrm{H}_{2} \quad \mathrm{H}_{3} \mathrm{H}_{4} \mathrm{H}_{5}$ \\
\hline \multirow{4}{*}{$\begin{array}{l}\text { Ratio of flutter } \\
\text { Oil pressure difference }\end{array}$} & 0.18 & $(0,0,0.1,0.53,0)$ \\
Actuat & 0.21 & $(0,0,0,0.83,0)$ \\
ors & between in and out & & \\
& Down oil pressure of oil & 0.23 & $(0,0,0.1,0.53,0)$ \\
& motor & 0.19 & $(0,0,0.1,0.53,0)$ \\
& Worn scraps in oil & 0.11 & $(0,0,0,0.73,0)$ \\
& Flux warp of valve & 0.08 & $(0,0,0.1,0.53,0)$ \\
\hline \multirow{3}{*}{ EH oil } & Power attenuation time & EH oil pump swing & 0.24 & $(0,0,0.1,0.53,0)$ \\
system & EH oil pump current & 0.27 & $(0,0,1,0,0)$ \\
& Oil temperature & 0.23 & $(0,0,0,0.76,0)$ \\
& EH oil main pipe pressure & 0.26 & $(0,0,0.65,0,0)$ \\
\hline Protect & OPC oil pressure & 0.5 & $(0,0,0,0.6,0.1)$ \\
ion sys & AST oil pressure & 0.5 & $(0,0,0,0,1)$ \\
\hline
\end{tabular}

Put above dada into the iterative formula, the condition analysis results of subsystems and system can be gained as follow:

Tab.8 Condition analysis results

\begin{tabular}{|c|c|c|c|}
\hline System & Weight & $\begin{array}{lllll}H_{1} & H_{2} & H_{3} & H_{4} & H_{5}\end{array}$ & $\begin{array}{l}\text { Preference } \\
\text { degree }\end{array}$ \\
\hline Actuators & 0.46 & $(0,0,0,0.29,0.57)$ & 0.686 \\
\hline EH oil system & 0.28 & $(0,0,0.33,0.19,0)$ & 0.076 \\
\hline Protection system & 0.26 & $(0,0,0.032,0.41,0)$ & 0.164 \\
\hline DEH system & - & $(0,0,0.06,0.23,0.22)$ & 0.312 \\
\hline
\end{tabular}

The above data indicate that the three subsystems are in "best", "general" and "good" condition respectively, and the whole DEH system is in "good" condition. Analyzing the preference degrees of the three subsystem, the EH oil system is in relatively poor condition. Based on this condition analysis, caution should be exercised and measure be taken in appropriate opportunity.

the condition analysis results can denote the system condition more accurately, and provide an effective support for next maintenance decision

\section{ACKNOWLEDGEMENTS}

The project is supported by Hebei science and technology department (15273727), and the Foundation of Hebei University of Science and Technology (XI201504)

\section{REFERENCES}

[1] Yujiong Gu, Yuliang Dong, Kun Yang, "Synthetic evaluation on conditions of equipment in power plant based on fuzzy judgment and RCM analysis", Proceedings of the CSEE, Vol.24, No.6, pp.189-194, June 2004.

[2] Gilchrist W, "Modelling failure modes and effects analysis", International Journal of Quality Reliability management, Vol. 10, No. 5, pp. 16-23, May 1993

[3] Chang C L, Wei C C, Lee Y H, "Failure mode and effects analysis using fuzzy method and grey method", Kybernetes, Vol. 28, No. 9, pp. 1072-1080, Sep.1999.

[4] Klir GJ, Yuan B, Fuzzy sets and fuzzy logic: theory and application, New Jersy: Prentice-Hall, 1995

[5] Kuusela H, Spence MT, Kanto AJ, "Expertise effects on pre-choice decision processed and final outcomes: a protocol analysis”, Eur J Market, Vol.32, No.5/6, pp.559-576, Jane. 1998.

[6] Bowles J B, Pelaez E C, "Fuzzy logic prioritization of failures in a system failure mode, effects and criticality analysis", Reliability Engineering and System Safety, Vol. 50, No. 2, pp. 203-213, Oct.1995.

[7] Zeng Huanglin, Intelligent computing, Chong qing university, Chong qing, 2004. 